
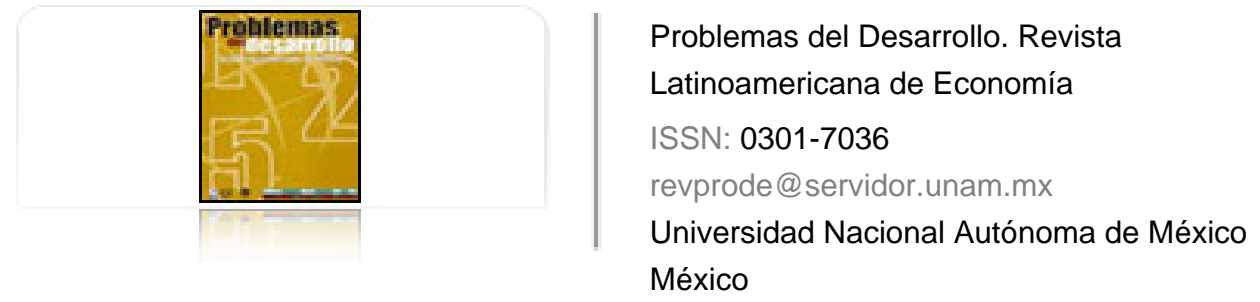

Pressman, Steven

La clase media en países latinoamericanos

Problemas del Desarrollo. Revista Latinoamericana de Economía, vol. 42, núm. 164, enero-marzo, 2011, pp. 127-152

Universidad Nacional Autónoma de México

Distrito Federal, México

Disponible en: http://www.redalyc.org/articulo.oa? $\mathrm{id}=11819774006$

- Cómo citar el artículo

- Número completo

- Más información del artículo

- Página de la revista en redalyc.org

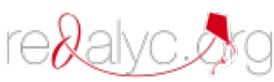

Sistema de Información Científica

Red de Revistas Científicas de América Latina, el Caribe, España y Portugal Proyecto académico sin fines de lucro, desarrollado bajo la iniciativa de acceso abierto 


\title{
LA CLASE MEDIA EN PAÍSES LATINOAMERICANOS
}

\author{
Steven Pressman*
}

Fecha de recepción: 11 de octubre de 2010. Fecha de aceptación: 10 de diciembre de 2010.

\section{RESUMEN}

En este artículo se utiliza el Estudio de Luxemburgo sobre Ingresos (ELI) para analizar la dimensión de la clase media en varios países latinoamericanos. Uno de los principales hallazgos es que a mediados de la primera década de este siglo, el tamańo de la clase media en América Latina no parecía depender de factores demográficos. Un segundo hallazgo es que, en contraste con la mayor parte de los países desarrollados, las políticas gubernamentales de impuestos y gastos influyen poco en el aumento del tamaño de la clase media en los países latinoamericanos de la muestra. Por último, al igual que en el mundo desarrollado, los factores del mercado laboral no ejercen gran efecto en el tamaño de la clase media en Latinoamérica, siendo las principales excepciones Brasil y México, donde el empleo parece incrementar el tamaño de la clase media.

Palabras clave: Clase media, distribución del ingreso, Estudio de Luxemburgo sobre el Ingreso (ELI), Latinoamérica, política de impuestos y gastos.

\section{THE MIDDLE CLASS IN LATIN AMERICAN COUNTRIES}

\section{Summary}

This paper uses the Luxembourg Income Study (LIS) to analyze the dimension of the middle class in various Latin American countries. One of its major findings is that during the first decade of this century, the size of the middle class in Latin America did not appear to depend on demographic factors. A second finding is that, in contrast with the majority of developed countries, government income and expenditure policies had little influence on increasing the size of the middle class in the Latin American countries in the sample. Finally, as in the developed world, labor market factors have little influence on the size of the middle class in Latin America, the main exceptions being Brazil and Mexico, where employment seems to increase the size of the middle class.

Key words: Middle class, income distribution, Luxembourg Income Study (LIS), Latin America, income and expenditure policy.

* Profesor-investigador del Departamento de Economía y Finanzas de la Universidad de Monmouth. Correo electrónico pressman@monmouth.edu. Traducción al español por Alma Chapoy Bonifaz, Instituto de Investigaciones de la UNAM. 


\section{LA CLASSE MOYENNE DANS LES PAYS LATINO-AMÉRICAINS Résumé}

Cette étude recourt à l'Étude sur les revenus du Luxembourg (ERL) pour analyser la dimension de la clase moyenne dans plusieurs pays latino-américains. Une des principales découverte est qu'au milieu de la première décennie de ce siècle, la taille de la classe moyenne en Amérique latine ne semblait pas dépendre de facteurs démographiques. Une deuxième découverte est que, à la différence de ce qui se passe dans la plupart des pays développés, les politiques gouvernementales d'impôts et dépenses influent peu pour augmenter la taille de la classe moyenne dans les pays latino-américains de l'échantillon examiné. Enfin, de même que dans le monde développé, les facteurs du marché du travail n'ont pas un grand effet sur la taille de la classe moyenne en Amérique latine, les principales exceptions étant le Brésil et le Mexique où l'emploi semble accroître la taille de la classe moyenne.

Mots clés : classe moyenne, distribution du revenu, Étude sur les revenus du Luxembourg (ERL), Amérique latine, politique d'impôts et dépenses

\section{A CLASSE MÉDIA NOS PAÍSES LATINO-AMERICANOS \\ Resumo}

Este trabalho utiliza o Estudo de Luxemburgo sobre Ingressos (ELI) para analisar a dimensáo da classe média em vários países latino-americanos. Um dos principais resultados encontrados é que a mediados da primeira década deste século, o tamanho da classe média em América Latina não pareceria depender de fatores demográficos. Um segundo resultado seria que, em contraste com a maior parte dos países desenvolvidos, as políticas governamentais de impostos e gastos influenciam pouco em aumentar o tamanho da classe média nos países latino-americanos da mostra. Por último, tal qual observado no mundo desenvolvido, os fatores do mercado laboral não exercem grande efeito no tamanho da classe media em América Latina, sendo as principais exceçóes Brasil e México, onde o emprego parece incrementar o tamanho da classe média.

Palavras-chave: Classe média, distribuição de renda, Estudo de Luxemburgo sobre Ingressos (ELI), América Latina, política de impostos e gastos.

\section{拉美国家的中产阶级}

摘要

本研究利用卢森堡收入研究（股票挂钩票据），分析了在拉美国家中产 阶级的规模。主要发现之一是, 在本世纪第一个十年中期, 在拉美中产 阶级的规模似乎还不取决于人口因素。第二个发现是, 与大多数发达国 家不同的是，政府的税收和支出政策对于中产阶级的规模影响不大。最 后，跟发达国家一样的是劳动力市场因素对中产阶级的规模影响不大。 主要的例外是巴西和墨西哥, 在此两国就业似乎会增加中产阶级的规 模。

关键词：中产阶级的收入分配，卢森堡收入（股票挂钩票据），拉丁美 洲, 税收和支出政策。 


\section{INTRODUCCIÓN}

Aquí me propongo extender mi anterior trabajo sobre cómo se comporta la clase media en diferentes países. Pressman $(2007,2010)$ proporciona una definición de "clase media" así como una estimación del tamaño de la misma en varias naciones, principalmente en países occidentales desarrollados. Esos documentos sostienen que las diferencias en la proporción de las familias de clase media, en varias naciones y a lo largo del tiempo, se deben principalmente a las políticas gubernamentales de impuestos y gastos. Mientras más progresivo sea el sistema fiscal nacional, y más generosos y amplios sean los programas de gasto gubernamental, mayor es la proporción de familias de clase media en una nación.

En este trabajo utilizo la Base de Datos del Estudio de Luxemburgo sobre Ingresos (ELI), una base de datos internacional que contiene amplia información socio-demográfica y acerca del ingreso. ${ }^{1}$ Esa base de datos se centra en años particulares, llamados ondas. Cada onda es de alrededor de cinco años; la onda 1 empieza a principios de los años ochenta. Los datos más recientes corresponden a la onda 6, y cubren el primer lustro de este siglo.

Hasta hace poco, México era el único país latinoamericano que aparecía en la base de datos del ELI. Esto cambió en el verano de 2009, cuando el ELI hizo pública la base de datos de la onda 6 , que incluía otras cinco naciones de América Latina (Brasil, Colombia, Guatemala, Perú y Uruguay). Junto con los datos anteriormente disponibles para México, esta nueva serie de datos proporciona una oportunidad única para evaluar el tamaño de la clase media en países de América Latina y compararla con la de los países desarrollados.

Esto es importante por varias razones. Aristotle (1932: Book IV) notó primero que las comunidades políticas con una gran clase media estaban bien gobernadas y la sociedad no estaba dominada por ninguno de los extremos de ingresos. Más recientemente, Thurow (1985) expuso que "se necesita una clase media saludable para tener una democracia saludable", pues la inquietud social se incrementa cuando los ingresos y el pueblo se polarizan. Barro (1999) proporciona apoyo empírico para este punto de vista: hay más probabilidad de que los países sean democráticos, mientras más alta sea la participación del ingreso

1 http://www.lisproject.org/techdoc.htm (multiple countries; accessed 29 August 2009 through 28 August 2010). Los interesados en más información acerca de la base de datos del ELI pueden consultar varios excelentes resúmenes (Smeeding et al, 1985; Smeeding, Rainwater, Buhmann \& Schmays 1988) o la página del eLI en www.lisproject.org. 
que va a las familias de clase media, aunque admite que la relación causal puede ir en ambas direcciones.

Hay también razones psicológicas por las cuales es importante una gran clase media. Alcanzar un nivel de vida de clase media, va acompañado de sentimientos de éxito y realización personal. Como Malthus (1803: 594) señaló en su segundo Ensayo sobre población, "Nuestras expectativas mejor fundadas de un incremento en la felicidad de la sociedad humana en su conjunto, se basan en la perspectiva de un incremento en las proporciones relativas de las partes medias".

El optimismo psicológico es probable que se extienda a optimismo económico, al llevar a más consumo, más inversión y más rápido crecimiento económico. Además, el solo hecho de que vaya más dinero a la clase media, debe conducir a mayor consumo y mayor crecimiento, pues los pobres tienen dinero para gastar y los ricos tienen demasiado dinero para gastar. Landes (1998: 217-21) identifica una creciente clase media como una de las principales razones por la que Gran Bretańa fue el primer país del mundo en industrializarse. Con una perspectiva más amplia, Adelman \& Morris (1967) seńalan que fue la creciente clase media lo que impulsó el desarrollo económico en toda Europa occidental.

Es probable que una robusta clase media sea tan importante para las naciones latinoamericanas, como lo fue en Europa. Estache \& Leipziger (2009) consideran que la distribución del ingreso es más importante para los países menos desarrollados porque una creciente clase media conduce a un mayor desarrollo económico, y no solamente a crecimiento económico, y esto también constituye una fuerza que impulsa la democracia y el apoyo a políticas económicas que ayudan tanto a las familias de clase media como a las de bajos ingresos. Easterly (2001) proporciona algún apoyo empírico a esto. Además, es bien sabido que la distribución del ingreso en Latinoamérica es la más desigual del mundo (Galbraith, 2002; Krozeniewicz \& Smith, 2000), y los programas gubernamentales tienden a ser más bien de naturaleza regresiva (Goldstein $\&$ Estache 2009).

En este documento se mide el tamaño de la clase media en algunos países latinoamericanos en los primeros cinco años de este siglo y se busca identificar algunos determinantes del tamaño de la clase media en estas naciones. Se empieza por definir a la "clase media", y por estimar su tamaño en algunas naciones de América Latina. Luego se evalúa el papel de los factores socio-demográficos, de las políticas fiscales gubernamentales, y de algunos factores del mercado laboral, en el desarrollo de una gran clase media en esos países. El Apéndice contiene información sobre las fuentes originales nacionales. 


\section{2. ¿QUIÉNES CONSTITUYEN LA CLASE MEDIA?}

Desafortunadamente, no hay definición económica oficial de "clase media", y ninguna definición que sea aceptable para la mayoría de los científicos sociales. Este documento emplea la sugerida por Birdsall et al (2000), Pressman (2007, 2010), y Thurow (1985), de que las familias de clase media son aquéllas cuyos ingresos están en la parte media de la distribución del ingreso nacional. En particular, una familia se considera de clase media si, y sólo si, su ingreso familiar disponible ajustado se sitúa entre 75 y $125 \%$ de la media ajustada del ingreso familiar disponible. Esta "relativa definición" de clase media es justificada en varios estudios que muestran que después de que se alcanza un cierto nivel de ingreso, los ingresos relativos le importan más a la gente (Frank, 2007; Layard, 2005; Luttmer, 2005), y por el trabajo de muchos académicos que han sostenido que las personas son seres sociales (Fuchs, 1967; Easterlin, 2001; Sen, 1999: 71) que continuamente se comparan unos con otros. En este trabajo necesitamos definiciones relativas y no definiciones absolutas de "clase media", "pobreza" y "bienestar humano".

Para medir el bienestar económico necesitamos ajustar el ingreso familiar tomando en cuenta las diferencias en el tamaño de las familias. Por ejemplo, en EUA un ingreso de $\$ 24,000$ puede sostener a un individuo razonablemente bien. En 2009, hubiera representado más del doble del ingreso de una persona en el nivel de pobreza. Pero para una familia de cinco miembros, un ingreso de $\$ 24,000$ proporciona a cada uno sólo $\$ 4,800$ en promedio. Con esto no se puede tener el mismo estilo de vida que si $\$ 24,000$ fueran para un solo individuo; de hecho, según la Oficina del Censo de EUA, en 2009, una familia de cinco integrantes tendría que ser considerada pobre con ese ingreso.

Una forma de manejar los diferentes tamaños de las familias es tratar igual las necesidades de ingreso de todos los miembros de la familia y considerar el ingreso per cápita. Pero esto ignora las economías de escala que surgen de vivir juntos. Dos personas gastan menos viviendo juntas que separadas, y tendrán un nivel de vida más alto que dos individuos que viven solos con el mismo ingreso combinado. Lo que necesitamos es alguna base intermedia entre suponer que no hay ese tipo de economías de escala y suponer que el tamaño de la familia es importante para su nivel de vida.

Por ello, ajustamos los ingresos familiares usando las recomendaciones de la OCDE (1982) respecto a escalas de equivalencia para diferentes tamaños de familias. De acuerdo con esta norma, los requerimientos de ingreso de los niños equivalen a $50 \%$ de los del jefe de familia, y las necesidades de ingreso de los otros adultos de la familia son de $70 \%$ de los requerimientos del jefe de familia. 
Esto se acerca mucho a los ajustes familiares implícitos o escala de equivalencias en la serie de líneas de la pobreza desarrollada por Mollie Orshanksy (1965, 1969) cuando ella estableció las líneas oficiales de pobreza en EUA para diferentes tamańos de familias. Como las líneas de la pobreza de Orshansky provienen de fuentes de consumo alimenticio y gastos, en diferentes familias, este enfoque proporciona una buena base empírica para usar las normas de la OCDE al ajustar el ingreso considerando el tamaño de la familia. Otras fórmulas de ajuste han sido sugeridas y probadas, y los estudios han encontrado que la diferencia es poca con los amplios resultados que se obtienen al usar el ELI (Smeeding, Buhmann \& Rainwater, 1988); sin embargo, obviamente, las cifras reales serán diferentes.

\section{EL TAMAÑo DE LA CLASE MEDIA EN LATINOAMÉRICA}

El Cuadro 1 proporciona información sobre el tamaño de la clase media para la muestra de países latinoamericanos. Considerando nuestra principal definición (el ingreso familiar disponible ajustado entre 75 y 125\% de la media ajustada del ingreso familiar), $21 \%$ de las familias pueden considerarse como clase media. Uruguay tiene con mucha ventaja la mayor clase media, pues casi $27 \%$ de las familias caen en esa categoría. Le sigue Colombia con 22.6\%. El resto de nuestros países se agrupan alrededor de 19\%. Con fines comparativos, para la onda 6 de las bases de datos del ELI, la clase media en los países desarrollados es, en promedio, del doble: $40 \%$ de todas las familias. En las naciones escandinavas (Dinamarca, Noruega y Suecia), se aproxima a 50\% de la población total. En Canadá y euA, Pressman (2010) estima que el tamaño de la clase media es de 35 y $29 \%$, respectivamente.

En general, las cifras del Cuadro 1 son coherentes con las que conocemos de otras fuentes acerca de estos países y su distribución del ingreso. Las estadísticas sucintas del ELI acerca de la distribución del ingreso para las seis naciones del cuadro, ${ }^{2}$ sitúan a Uruguay, por mucho, como el país con la distribución del ingreso más equitativa con base en diferentes medidas de distribución, incluyendo el coeficiente de Gini y el de Atkinson, y la ratio de ingreso recibido por alguien en el $90^{\circ}$ percentil en relación a alguien en el $50^{\circ}$ percentil de la distribución del ingreso. En el otro extremo, es ampliamente reconocido (y confirmado

2 Disponibles en el sitio del ELI www.lisproject.org. 
por las estadísticas del ELI) que Brasil tiene una de las más inequitativas distribuciones del ingreso en Latinoamérica y en el mundo entero; y como lo muestra el Cuadro 1, ese país tiene la clase media más pequeńa de las seis naciones latinoamericanas de la muestra.

Cuadro 1. Familias de la clase media (como porcentaje de todas las familias)

Definiciones alternativas de la clase media

\begin{tabular}{lcccc}
\hline \multicolumn{1}{c}{ País } & $\begin{array}{c}\text { Principal } \\
\text { definición }\end{array}$ & D2 & D3 & D4 \\
\hline Brasil & $18.8 \%$ & $23.5 \%$ & $26.7 \%$ & $29.0 \%$ \\
Colombia & $22.6 \%$ & $29.0 \%$ & $33.9 \%$ & $38.7 \%$ \\
Guatemala & $19.1 \%$ & $26.8 \%$ & $32.5 \%$ & $36.3 \%$ \\
México & $19.7 \%$ & $24.6 \%$ & $28.1 \%$ & $30.7 \%$ \\
Perú & $19.5 \%$ & $26.5 \%$ & $32.3 \%$ & $36.9 \%$ \\
Uruguay & $26.8 \%$ & $35.5 \%$ & $41.6 \%$ & $46.3 \%$ \\
Promedios (No ponderados) & $21.1 \%$ & $27.7 \%$ & $32.5 \%$ & $36.3 \%$ \\
\hline
\end{tabular}

Fuente: Cálculos del autor con base en el Estudio de Luxemburgo sobre el ingreso.

Nota: La principal definición es la media ajustada del ingreso familiar $\pm 125 \%$ de la media; D2 = 75\% de la media $>$ media y $<150 \%$ de la media; D3 = 75\% de la media $>$ media $<175 \%$ de la media; $\mathrm{D} 4=75 \%$ de la media $>$ media $<200 \%$ de la media.

Como no hay definición generalmente aceptada de "clase media" y como la elección de cualquier rango de ingreso será arbitraria en alguna medida, el Cuadro 1 también proporciona un análisis de sensibilidad de nuestras estimaciones. Las últimas columnas emplean tres definiciones adicionales de clase media, relacionadas con el ingreso, (D2) ingreso familiar ajustado entre 75 y $150 \%$ de la media ajustada del ingreso disponible, (D3) ingreso familiar ajustado entre 75 y $175 \%$ de la media ajustada del ingreso disponible, y (D4) ingreso familiar entre 75 y $200 \%$ de la media ajustada del ingreso disponible.

La definición D2 es importante porque en un análisis reciente de la clase media en EuA, el Pew Research Center (2008) sostuvo que éste era el rango más apropiado para identificar a las familias de clase media y usó este rango en su estudio acerca de la clase media. La definición D4 es significativa porque proporciona un rango de ingreso para la clase media, cercano a lo que realmente informaron las familias de EuA cuando se les preguntó acerca de los niveles de ingreso necesarios para incluir a una familia de cuatro miembros en la clase media (Pew 
Research Center, 2008; Cashell, 2008). Sin embargo, D4 tiene algunos inconvenientes. Incluir a las familias con $200 \%$ del ingreso medio como parte de la clase media, significa que sólo alrededor de 3\% de la población de EuA pertenece a la clase rica o alta. Además, este límite superior está sustancialmente por encima del que la mayoría de los académicos considera como el que proporciona los niveles y estilos de vida de la clase media. No obstante, D4 refleja los puntos de vista populares de la clase media; y puede tener más sentido usar esta definición en las naciones de Latinoamérica. Al usar D4, la clase más alta constituye de 10 a $20 \%$ de las familias en la mayoría de nuestras seis naciones, y en Guatemala y Perú la clase más alta se aproxima a $25 \%$ de todas las familias. Finalmente, la definición D3 se incluye como un punto intermedio entre lo sugerido por Pew y el rango sugerido por las fuentes empíricas de EUA.

Las buenas noticias son que estas cuestiones de definición no significan una diferencia sustancial. Pressman (2010) muestra que el rango de ingreso real para definir "clase media" importa muy poco en las naciones desarrolladas, y cualquier decisión sobre dónde trazar la línea no modifica los resultados empíricos obtenidos de adoptar cualquier rango de ingreso. Por supuesto, el tamaño estimado de la clase media se incrementa cuando se incluyen rangos de ingreso más y más grandes en nuestra definición. Sin embargo, el tamaño de la clase media varía según la nación de que se trate en el mismo patrón para todos los rangos de ingreso. En el mundo desarrollado, los países con una clase media relativamente grande en nuestra principal definición, también tienen una clase media relativamente grande en las definiciones alternativas. Las naciones escandinavas siempre tienen la clase media más grande; los países anglosajones, la más pequeña; y las naciones de Europa continental (Francia, Alemania, Italia y Luxemburgo) caen en el centro.

Para los países latinoamericanos, nuestros resultados son similares. En cada una de nuestras definiciones, Uruguay está mejor, con una sustancial diferencia, y le sigue Colombia. En el otro extremo, Brasil siempre es el peor de los seis países. Y en cada caso, Guatemala, México y Perú se sitúan siempre en el centro.

Esto indica que nuestros principales resultados no parecen depender de cuestiones relacionadas con la definición. Para la mayor parte de los rangos razonables de ingreso, los países con una clase media relativamente grande según una definición, también tienen una clase media relativamente grande usando otras definiciones. Los países que están mal, usando una definición, también lo están con otras definiciones.

Las siguientes tres secciones tratan algunos de los principales factores que podrían conducir a una clase relativamente grande en algunos países y a una clase media relativamente pequeña, en otros. Primero examino algunos posibles 
determinantes socio-demográficos de la clase media. Luego considero la política fiscal gubernamental y cómo ésta afecta el tamaño de la clase media. Finalmente, me refiero a los factores del mercado laboral que promueven u obstaculizan a la clase media.

\section{LOS FACTORES SOCIO-DEMOGRÁFICOS Y LA CLASE MEDIA}

El Cuadro 2 presenta estimaciones sobre el tamaño de la clase media en nuestros países latinoamericanos, basadas en varias características socio-demográficas de las familias. Por muchas razones, diversos factores socio-demográficos podrían explicar la cambiante distribución del ingreso y los cambios en el tamaño de la clase media.

Cuadro 2. Familias de clase media por características socio-demográficas seleccionadas (como porcentaje de todas las familias)

\begin{tabular}{lccccccc}
\hline \multicolumn{1}{c}{ País } & $\begin{array}{c}\text { Todas las } \\
\text { familias }\end{array}$ & $\begin{array}{c}\text { De edad } \\
\text { avanzada }\end{array}$ & $\begin{array}{c}\text { Familias en } \\
\text { la primera } \\
\text { edad }\end{array}$ & $\begin{array}{c}\text { Familias } \\
\text { encabezadas } \\
\text { por mujeres }\end{array}$ & $\begin{array}{c}\text { Parejas } \\
\text { casadas }\end{array}$ & $\begin{array}{c}\text { Familias } \\
\text { con niños }\end{array}$ & $\begin{array}{c}\text { Familias } \\
\text { que viven } \\
\text { en áreas } \\
\text { urbanas }\end{array}$ \\
\hline Brasil & $18.8 \%$ & $22.0 \%$ & $18.2 \%$ & $19.1 \%$ & N.A. & $14.9 \%$ & $20.2 \%$ \\
Colombia & $22.6 \%$ & $20.9 \%$ & $22.8 \%$ & $19.1 \%$ & $23.3 \%$ & $25.0 \%$ & $21.4 \%$ \\
Guatemala & $19.1 \%$ & $18.8 \%$ & $18.6 \%$ & $20.7 \%$ & $18.3 \%$ & $20.1 \%$ & $19.7 \%$ \\
México & $19.7 \%$ & $15.4 \%$ & $20.3 \%$ & $20.6 \%$ & $19.8 \%$ & $19.2 \%$ & $24.1 \%$ \\
Perú & $19.5 \%$ & $17.0 \%$ & $20.2 \%$ & $20.1 \%$ & $18.5 \%$ & $21.2 \%$ & $22.2 \%$ \\
Uruguar & $26.8 \%$ & $31.2 \%$ & $23.8 \%$ & $27.1 \%$ & $27.0 \%$ & $23.4 \%$ & N.A. \\
Promedios no & $21.1 \%$ & $20.9 \%$ & $20.7 \%$ & $21.1 \%$ & $21.4 \%$ & $20.6 \%$ & $21.5 \%$ \\
ponderados & & & & & & & \\
\hline
\end{tabular}

Fuente: Cálculos del autor con base en el ell

Nota: La clase media se define como hogares entre 75\% y 125\% de la media ajustada de los ingresos familiares. Las familias en la primera edad son aquellas cuyo jefe de familia tiene entre 35 y 50 años; las familias de edad avanzada son los hogares encabezados por personas de 65 años o más. En el caso de México y Perú lo urbano esta definido por la población

Es bien sabido que, en promedio, los ingresos dependen de la edad. Trabajadores jóvenes e inexpertos, por lo general reciben menos paga que otros trabajadores. Los ingresos suben con la edad y tienden a alcanzar su cima alrededor de los cincuenta años; poco después, los ingresos caen con la edad. Consecuentemente, si cambia la distribución por edad de la población 
trabajadora, habrá modificaciones en la distribución del ingreso y posiblemente en el tamaño de la clase media. Si una gran parte de las familias está en sus años más productivos, la igualdad en el ingreso se incrementa y habrá más familias de clase media; por el contrario, si gran parte de las familias está encabezada por muy jóvenes o por viejos, la desigualdad en el ingreso se incrementa y habrá menos familias de clase media.

Los acuerdos de vivir juntos pueden también afectar el tamaño de la clase media. Como las mujeres ganan menos que los hombres, una mayor parte de hogares encabezados por mujeres incrementaría la desigualdad en el ingreso y reduciría el tamaño de la clase media. Al contrario, más parejas casadas, incrementarían el porcentaje total de hogares de clase media. Sin embargo, donde las responsabilidades de criar a los niños limitan las posibilidades de empleo, las mujeres no pueden trabajar tanto como lo harían en hogares sin niños, y sería más difícil obtener el estatus de clase media.

Finalmente, el desarrollo económico por lo general se relaciona con una creciente clase media. Históricamente, una buena fracción de ésta surge de la migración del campo a la ciudad. Las familias ocupadas en la agricultura de subsistencia se mudan a las grandes ciudades donde obtienen ingresos más altos. A medida que las familias empiezan a moverse a las áreas urbanas, la desigualdad en el ingreso empieza a incrementarse debido a los ingresos crecientes en las zonas urbanas.

Para examinar el efecto de los factores socio-demográficos en el tamaño de la clase media, consideramos varios subgrupos clave de la población total. Con la finalidad de facilitar el seguimiento de estas comparaciones, empezamos por repetir los principales resultados del Cuadro 1, el porcentaje de los hogares que son de clase media.

El Cuadro 2 muestra, con algunas excepciones, que los factores socio-demográficos no parecen afectar el tamaño de la clase media. El porcentaje de hogares de clase media encabezados por personas mayores de 60 ańos, el porcentaje de hogares de clase media encabezados por personas cuyas edades fluctúan entre 35 y 50 ańos, el porcentaje de hogares de clase media encabezados por mujeres, el porcentaje de parejas casadas de clase media, el porcentaje de hogares de clase media con niños, y el porcentaje de familias de clase media que viven en áreas urbanas, no son muy diferentes de las cifras agregadas presentadas en la columna 1. Estos resultados son coherentes con los resultados en las naciones desarrolladas: en la mayoría de los casos, los factores socio-demográficos parecen importar muy poco para el tamańo de la clase media (Pressman, 2007, 2010). En el mundo desarrollado, en muy pocos países las características socio-demográficas parecen ser un factor determinante del estatus de clase media, y en pocos, los 
cambios demográficos pueden explicar buena parte de las modificaciones en el tamaño de la clase media.

Sin embargo, conviene destacar dos diferencias entre las naciones desarrolladas de occidente y los países latinoamericanos de la muestra. Primera, en contraste con aquéllas, donde las familias con niños es más probable que sean de clase media (ver Pressman, 2010), en las naciones de América Latina, las familias con niños tienen iguales probabilidades de ser de clase media que el resto de las familias. Una posible explicación de esto, que será explorada más adelante, es que la política gubernamental ayuda más a las familias con niños en las naciones desarrolladas. Segunda, el Cuadro 2 muestra que los hogares latinoamericanos encabezados por personas de mayor edad es más probable que sean de clase media en Brasil y Uruguay, pero esto es menos probable en México y Perú. También esto puede deberse a la política gubernamental: en algunas naciones existe un generoso sistema público de pensiones y en otras no.

Otro factor socio-demográfico que puede afectar el tamaño de la clase media, es el hecho de que la esposa trabaje; en particular, el que un hogar sea de clase media puede depender de las ganancias de la esposa y de su nivel de ingreso en relación al ingreso de su esposo. Si los hombres de ingreso alto, probablemente se casan con mujeres de ingreso alto, el resultado será una mayor desigualdad y más pocos hogares de clase media, puesto que si en un matrimonio los dos ganan un buen salario, serán impulsados a la clase más alta. Esto se ha convertido en una cuestión polémica entre los académicos que consideran el efecto de las ganancias de la esposa sobre la desigualdad del ingreso en EUA (Burtless, 1999; Cancian \& Reed, 1998, 2001; Esping-Andersen, 2007). Varios estudios han encontrado una creciente correlación entre las ganancias de marido y mujer a lo largo del tiempo en EUA (Shorrocks, 1983; Lerman \& Yitzhaki, 1985; Karoly \& Burtless, 1995). Esto significa que los hombres de alto salario es más probable que se casen con mujeres de altos ingresos, lo que incrementa la desigualdad en general. Por otra parte, Gottschalk \& Danziger (2005) muestran que los cambios en las horas de trabajo de las mujeres y la desigualdad en las ganancias de éstas a lo largo del tiempo, han hecho más equitativa la distribución del ingreso en EUA, y Cancian \& Schoeni (1999) encontraron que en la mayoría de los países desarrollados, las ganancias de las esposas tienden a mitigar la desigualdad entre las familias de parejas casadas. Cancian \& Reed (1998), en lo que quizá sea la mejor síntesis de esta tan diversa literatura, expresan que si las ganancias de las esposas han incrementado o disminuido la equidad en EUA o si no tienen efecto alguno sobre la desigualdad del ingreso, depende de cómo se mida.

El Cuadro 3 hace un primer intento de examinar esta cuestión en los seis países latinoamericanos. Allí se considera cómo las ganancias de la esposa afectan 
el tamaño de la clase media en las naciones latinoamericanas. Para analizar esto empezamos por calcular el porcentaje de familias de clase media constituidas por una pareja no de edad avanzada. Luego, al ingreso familiar le restamos las ganancias de la esposa y recalculamos el porcentaje que representan las familias de clase media.

Cuadro 3. Efecto de las ganancias de las esposas sobre el tamaño de la clase media

\begin{tabular}{lcc}
\hline \multicolumn{1}{c}{ País } & $\begin{array}{c}\text { \% de parejas casadas de la clase media } \\
\text { no de edad avanzada }\end{array}$ & $\begin{array}{c}\text { \% de parejas casadas no de edad } \\
\text { avanzada que son de clase media sin } \\
\text { ingresos de las esposas }\end{array}$ \\
\hline Brasil & $17.7 \%$ & $13.6 \%$ \\
Colombia & $24.0 \%$ & $26.8 \%$ \\
Guatemala & $18.7 \%$ & $19.8 \%$ \\
México & $20.5 \%$ & $18.7 \%$ \\
Perú & $19.2 \%$ & $20.9 \%$ \\
Uruguay & $25.4 \%$ & $23.2 \%$ \\
Promedios no ponderados & $20.9 \%$ & $20.5 \%$ \\
Canadá & $36.4 \%$ & $34.8 \%$ \\
EUA & $31.3 \%$ & $20.3 \%$ \\
\hline
\end{tabular}

Fuente: Cálculos del autor utilizando la base de datos del ell.

Nota: La clase media se define como las familias con entre 75 y 125\% de la media ajustada del ingreso familiar; no de edad avanzada significa que el jefe de familia tiene menos de 60 años.

*Para Brasil los datos están disponibles sólo por parejas de hogar que pueden o no estar casadas.

Con base en este ejercicio, las ganancias de las esposas parecen tener poco efecto sobre el tamaño de la clase media en nuestra muestra de seis naciones de América Latina. En Colombia, Guatemala y Perú, las ganancias de las esposas realmente reducen el tamaño de la clase media. En estos casos, lo más probable es que las ganancias de las esposas impulsen los ingresos al alza y ubiquen a las familias en la categoría de ingreso más alto y no que lleven a las familias de bajo ingreso a la clase media. Con fines comparativos, en la parte inferior del Cuadro 3, agregamos datos para las dos naciones desarrolladas de América: Canadá y EUA. EUA es único en cuanto a que las ganancias de las esposas parecen importar mucho para el estatus de clase media. De hecho, sin estas ganancias, EUA se parecería mucho a nuestras seis naciones latinoamericanas, en términos del tamańo de parejas casadas de clase media. A pesar de tener una mucho mayor clase 
media que los otros países del Cuadro 3, Canadá se parece más a las naciones de América Latina en un aspecto: las ganancias de las esposas influyen poco en llevar el ingreso de los hogares a los niveles de clase media. Mucho de esto puede deberse a la disponibilidad de beneficios gubernamentales en Canadá, que apoyan a las parejas casadas. Regresaremos a esto más adelante.

\section{POLÍ́tica gUbERNAMENTAL Y CLASE MEDIA}

Pressman (2007) sostiene que la política fiscal gubernamental es un importante determinante del tamaño de la clase media en todas las naciones desarrolladas; Pressman (2010) mostró que dos políticas específicas (asignaciones familiares y permisos familiares) incrementan de manera significativa el tamaño de la clase media en estas naciones y que esto es especialmente verdad en los hogares con niños. Las asignaciones familiares son pagos anuales a las familias para ayudarlas en la manutención de sus hijos. Virtualmente todos los países tienen algún tipo de política de asignaciones familiares o para los niños (Macinol, 1980; Vadakin, 1958, 1968). Las políticas de permisos familiares incluyen pagos a futuras madres que se ausentan de su trabajo regular, pagos por nacimiento, y pagos a padres de recién nacidos para que puedan permanecer en casa y atender a su bebé. La mayoría de las naciones desarrolladas tienen algún tipo de tales políticas, pero éstas no son tan comunes en las naciones menos desarrolladas y en caso de haberlas, son menos generosas.

Éstas son sólo dos de las muchas políticas fiscales empleadas en el mundo desarrollado para que las familias puedan disfrutar de los beneficios de un estilo de vida de clase media. Los beneficios de desempleo y el seguro por discapacidad garantizan que los problemas económicos de corto plazo o el problema de salud de largo plazo, no traerán como consecuencia una abyecta pobreza para la familia. Los programas gubernamentales de pensiones surgieron para impedir que los hogares de personas de edad avanzada cayeran en la pobreza, al obligarlos a ahorrar durante sus ańos productivos y al proporcionarles un ingreso fijo durante sus años de retiro. Estos pagos permiten a los hogares de personas de edad avanzada, seguir viviendo una existencia de clase media después de su jubilación. Por último, un sistema fiscal progresivo, especialmente donde los hogares de bajo ingreso pagan impuestos negativos, también ayuda a apoyar los ingresos y estilos de vida de la clase media en tiempos difíciles. Esta sección examina estas políticas gubernamentales en el contexto de los países latinoamericanos.

Para ello, seguimos el procedimiento empleado al examinar el efecto de las ganancias de las esposas sobre el tamaño de la clase media. Primero, consideramos 
el tamaño de la clase media en un subgrupo seleccionado de la población. Luego restamos las fuentes de ingreso gubernamental del ingreso familiar y recalculamos el porcentaje de familias que son de clase media.

Por supuesto, surge la pregunta de si el ingreso adicional proveniente del gobierno afecta la conducta individual. Por ejemplo, asignaciones infantiles pueden alentar a las familias a tener más hijos. Como es menos probable que las familias más grandes sean de clase media, al ignorar cambios en el comportamiento, hemos sobreestimado el tamaño de la clase media. En los países desarrollados, varios estudios han encontrado cambios en el comportamiento muy pequeños e insignificantes como resultado de esta política (Ermisch, 1988a, 1988b; Gauthier \& Hatzius, 1997). Además, puede haber efectos macroeconómicos derivados de las políticas gubernamentales que contrarresten cualquier cambio de comportamiento. Si las asignaciones infantiles incrementan el tamaño de la clase media y por tanto los gastos familiares, ello expandirá la economía, lo que además incrementará el tamaño de la clase media. Por esta razón, aquí suponemos que no hay cambios de comportamiento. En la medida en que las políticas gubernamentales conduzcan a cambios de comportamiento, y en la medida en que estos cambios excedan los efectos sobre el ingreso de estas políticas, nuestras estimaciones del efecto que estas políticas gubernamentales tienen sobre la clase media serán sesgadas al alza.

Lo que es verdad para las asignaciones infantiles, lo es también para las pensiones gubernamentales y para el seguro de desempleo. Estos beneficios pueden alentar a la gente a conseguir beneficios en vez de trabajar. De nuevo, nosotros ignoramos estos posibles efectos, suponiéndolos cercanos a cero. Tal supuesto es muy razonable en las naciones menos desarrolladas, ya que los niveles de beneficio son por lo general menores y las condiciones para recibir beneficios, más estrictas. Y, como en el caso de las asignaciones infantiles, el seguro de desempleo y las pensiones gubernamentales, incrementarían los gastos totales, el crecimiento económico, y de este modo, el tamaño de la clase media. Este efecto macroeconómico o efecto ingreso contrarrestaría cualquier efecto de sustitución microeconómica de estas políticas gubernamentales.

El Cuadro 4 muestra que, en nuestra serie de seis países latinoamericanos, las políticas gubernamentales no son efectivas para desarrollar una clase media nacional. Las asignaciones infantiles y los permisos familiares incrementan el porcentaje de familias con niños que son de clase media, pero sólo una fracción de un punto porcentual en Brasil y Uruguay, los únicos dos países para los cuales tenemos esos datos en el ELI.

Ambas naciones otorgan asignaciones infantiles sólo a familias de bajo ingreso. Por ejemplo, en Uruguay, las asignaciones familiares están disponibles sólo 
para familias cuyos ingresos mensuales no exceden de tres veces el salario mínimo. Como el salario mínimo era demasiado bajo en 2004, tres veces esa cantidad era aun menos de la mitad del salario mensual promedio. Es difícil con tan precarias asignaciones infantiles ( $8 \%$ del salario mínimo) aumentar el número de familias de clase media.

Cuadro 4. Papel de las políticas gubernamentales en la creación de ingresos para la clase media (\% de familias que son clase media)

\begin{tabular}{|c|c|c|c|c|c|c|}
\hline País & $\begin{array}{l}\text { Familias con } \\
\text { niños }\end{array}$ & $\begin{array}{c}\text { Familias con } \\
\text { niños menos } \\
\text { asignaciones } \\
\text { infantiles } \\
\text { y permisos } \\
\text { familiares }\end{array}$ & $\begin{array}{c}\text { No de edad } \\
\text { avanzada }\end{array}$ & $\begin{array}{l}\text { No de edad } \\
\text { avanzada } \\
\text { menos } \\
\text { desempleo e } \\
\text { incapacidad }\end{array}$ & $\begin{array}{l}\text { De edad } \\
\text { avanzada }\end{array}$ & $\begin{array}{c}\text { De edad } \\
\text { avanzada } \\
\text { menos } \\
\text { pensiones }\end{array}$ \\
\hline Brasil & $14.9 \%$ & $14.8 \%$ & $17.9 \%$ & $17.6 \%$ & $22.0 \%$ & $6.7 \%$ \\
\hline Colombia & $25.0 \%$ & N.A. & $23.1 \%$ & N.A. & $20.9 \%$ & $22.0 \%$ \\
\hline Guatemala & $20.1 \%$ & N.A. & $19.3 \%$ & N.A. & $18.8 \%$ & $20.9 \%$ \\
\hline México & $19.2 \%$ & N.A. & $20.5 \%$ & N.A. & $15.4 \%$ & $12.0 \%$ \\
\hline Perú & $21.2 \%$ & N.A. & $20.3 \%$ & $20.3 \%$ & $17.0 \%$ & $16.8 \%$ \\
\hline Uruguay & $23.4 \%$ & $23.0 \%$ & $24.6 \%$ & $24.4 \%$ & $31.2 \%$ & $9.6 \%$ \\
\hline $\begin{array}{l}\text { LDC Promedios } \\
\text { no ponderados }\end{array}$ & $20.6 \%$ & $18.9 \%$ & $21.0 \%$ & $20.8 \%$ & $20.9 \%$ & $14.7 \%$ \\
\hline Canadá & $38.4 \%$ & $36.1 \%$ & $33.7 \%$ & $31.7 \%$ & $41.6 \%$ & $15.8 \%$ \\
\hline EUA & $32.3 \%$ & $32.2 \%$ & $29.8 \%$ & $29.5 \%$ & $28.0 \%$ & $12.1 \%$ \\
\hline
\end{tabular}

Fuente: Cómputos del autor utilizando la base de datos del Ell.

Nota: De edad no-avanzada significa que el jefe de familia tiene menos de 60 años.

En contraste, Pressman (2010) encuentra que en las naciones desarrolladas que aplican tal política, las asignaciones infantiles incrementan en cerca de cinco puntos porcentuales la proporción de familias con niños que son clase media. Pero en América, estas políticas proporcionan poco apoyo y son relativamente poco efectivas en ayudar a que las familias alcancen el estatus de clase media. En Canadá, incrementan el tamaño de la clase media en 2.1 puntos porcentuales; en EUA, el incremento es de cero ya que EUA no tiene programa de asignaciones familiares. La parte inferior del Cuadro 4 toma en cuenta las políticas de permisos familiares así como las asignaciones infantiles en Canadá y EUA. Esta adición cambia poco los resultados provenientes sólo de asignaciones familiares. De las primeras columnas del Cuadro 4, y de datos similares para los países 
desarrollados, podemos concluir que una razón de que Latinomérica tenga una clase media más pequeña que la de los países desarrollados, son sus programas tan precarios de asignaciones infantiles.

Los seguros de desempleo y discapacidad no son mejores que las políticas de asignaciones familiares y de permisos familiares en las tres naciones para las cuales se tienen datos del ELI. En general, los pagos por desempleo y por incapacidad son pequeños en las naciones latinoamericanas y muchas familias (especialmente las que trabajan en el sector informal) no son elegibles para esos pagos (ECLAC, 2005; Lindert et al, 2006). Como en Brasil el otorgamiento del seguro de desempleo requiere una investigación para determinar quién tiene derecho o no a esa prestación, los individuos desempleados con un cónyuge que trabaja, es probable que no califiquen y que no permanezcan en la clase media como resultado de los beneficios de desempleo. Por ello, el incremento de los hogares de clase media debido a estas dos políticas, es apenas de una pequeña fracción de un punto porcentual.

Las últimas filas del Cuadro 4, comparan nuestras naciones de Latinoamérica con las naciones desarrolladas del continente americano. Los seguros de desempleo y de incapacidad en Canadá (un país relativamente tacaño en lo que concierne a beneficios gubernamentales) incrementan el tamańo de la clase media en 2 puntos porcentuales. EUA, la más mezquina de las principales naciones desarrolladas en estas cuestiones, lo hace sólo un poco mejor que los tres países latinoamericanos para los cuales tenemos datos. Así, parece que una segunda razón para una clase media pequeña en Latinoamérica son sus raquíticos programas de desempleo e incapacidad. En Brasil, el otorgamiento del seguro de desempleo exige una investigación previa; en consecuencia, es de poca ayuda a las familias que reciben dos ingresos, cuando sólo uno de los dos pierde su trabajo. Sin embargo, las ganancias de un solo adulto probablemente no basten para mantener el estatus de familia de clase media.

Únicamente en el caso de las pensiones, la política gubernamental en América Latina es de alguna ayuda. Las pensiones incrementan el tamaño de la clase media de más edad en varios países, y en algunos casos (Brasil y Uruguay) de manera significativa. En Brasil, los beneficios de jubilación equivalen a 70\% de las ganancias promedio, más otro $1 \%$ por cada año de contribución al sistema, con un pago máximo de $100 \%$ de los ingresos promedio. Con tan generosos beneficios, es relativamente fácil recibir al jubilarse una gran parte de los ingresos promedio y mantener en esa etapa el estatus de clase media.

También debe señalarse que en los casos de Colombia y Guatemala, las pensiones realmente reducen el tamaño de la clase media. Además, las diferencias de país a país son muy grandes. Una buena parte de estas diferencias probablemente surjan de diferencias nacionales en el porcentaje de la fuerza de trabajo 
empleada en los sectores informales que no son cubiertos por pensiones de jubilación. El conjunto de estos resultados son coherentes con una amplia literatura que encuentra que el grueso de los gastos sociales en Latinoamérica, especialmente en pensiones, no son efectivos para proteger a los pobres y favorecen a las familias que se encuentran en los dos quintiles de ingreso más altos (ECLAC, 2005; Lindert et al, 2006). Por ejemplo, de acuerdo con esta literatura, la mayor parte de los gastos en seguridad social van a los dos quintiles más altos, $80 \%$ en Colombia, y 50 \% en Brasil y Uruguay (eClaC, 2005: 144-6).

De nuevo, el contraste con las naciones desarrolladas es grande e importante. En Canadá y EUA, las pensiones son significativamente más efectivas para incrementar la proporción de familias de mayor edad de la clase media. En contraste con un incremento promedio de 6.2 puntos porcentuales en nuestros seis países latinoamericanos, el incremento promedio es de casi 16 puntos porcentuales en EUA y de casi 26 en Canadá. Y hay que recordar que la política gubernamental en Canadá y EUA es relativamente mala para promover y sostener una clase media, si se le compara con la de otras naciones desarrolladas de Occidente (ver Pressman, 2010).

Finalmente, nos referiremos al papel de la política fiscal sobre la clase media. Otra vez hemos de señalar que hay importantes limitaciones para realizar este análisis. La mitad de las series de datos del ELI para nuestras naciones latinoamericanas están en términos netos (excluyendo impuestos), y así no tenemos información acerca de los impuestos pagados por los diferentes hogares. El Cuadro 5 proporciona información sobre el efecto de los impuestos en el tamaño de la clase media para todas las series de datos con información sobre pago de impuestos hecho por las familias, junto con estimaciones comparables para Canadá y EUA.

Cuadro 5. Política fiscal y familias de clase media

\begin{tabular}{lcc}
\hline \multicolumn{1}{c}{ País } & $\begin{array}{r}\text { \% de familias de clase media con } \\
\text { base en el ingreso disponible }\end{array}$ & $\begin{array}{c}\text { \% de familias de clase media con } \\
\text { base en el ingreso bruto }\end{array}$ \\
\hline Brasil & $18.8 \%$ & $18.9 \%$ \\
Colombia & $22.6 \%$ & $22.5 \%$ \\
Guatemala & $19.1 \%$ & $18.9 \%$ \\
México & N.A. & N.A. \\
Perú & N.A. & N.A. \\
Uruguay & N.A. & N.A. \\
Promedios no ponderados & $20.2 \%$ & $20.1 \%$ \\
Canadá & $34.9 \%$ & $26.6 \%$ \\
EUA & $28.7 \%$ & $23.5 \%$ \\
\hline
\end{tabular}

Fuente: Cómputos del autor utilizando la base de datos del Ell. 
Como se observa, la política fiscal en Brasil, Colombia y Guatemala, cambia poco el tamaño de la clase media en estas naciones. Dicho cambio es de sólo una décima, o algo así, de un punto porcentual, esencialmente cero. El contraste con Canadá y EUA es enorme. En EUA, la política fiscal incrementa el tamaño de la clase media en más de cinco puntos porcentuales. En Canadá, el incremento es de más de ocho puntos porcentuales. En una muestra más grande de las principales naciones desarrolladas, Pressman (2010) encontró que la política fiscal (impuestos sobre el ingreso e impuestos obligatorios de seguridad social) incrementó el tamaño de la clase media en 7.5 puntos porcentuales. La política fiscal actuó principalmente reduciendo el ingreso de los hogares más ricos y llevando a éstos a la clase media. Pero hay que recordar que el dinero obtenido mediante un sistema fiscal progresivo sirvió para elevar el ingreso de otros hogares y proporcionar ingresos de clase media a muchas familias. De esta manera, en las naciones desarrolladas el efecto total de los impuestos en el tamaño de la clase media, es mucho mayor de lo que se presenta en el Cuadro 5.

\section{FACTORES DEL MERCADO LABORAL Y TAMAÑO DE LA CLASE MEDIA}

Del mismo modo que los factores socio-demográficos y la política gubernamental pueden afectar el tamaño de la clase media, las fuerzas del mercado laboral pueden influir sobre el tamaño de la clase media. Esta sección examina el efecto que algunas variables del mercado laboral tienen sobre el tamaño de la clase media.

Varios estudios llevados a cabo con datos de euA muestran que el tamaño de la clase media varía con el ciclo económico (e.g., Horrigan \& Haugen, 1988). Si el desempleo es un determinante clave del tamaño de la clase media, nosotros esperaríamos que el creciente desempleo en una nación conduciría a una caída en el tamaño de la clase media, ya que muchas familias perderían empleo e ingreso. Pressman (2007: 190-91) examinó esta cuestión en el nivel macroeconómico y encontró que los cambios en la tasa nacional de desempleo tenían poca correlación con los cambios en el tamaño de la clase media nacional. Una posible razón de ello es que los beneficios de desempleo compensan la pérdida de ingreso derivada del desempleo y mantienen a los hogares en la clase media. Otra posible razón es que amplias variables macroeconómicas, como la tasa nacional de desempleo, no puede capturar lo que está sucediendo en el nivel microeconómico.

Pressman (2010) examinó esto en un nivel más microeconómico. Usando microdatos del ELI acerca del número de perceptores de ingreso, analizó cuántos de los cambios en la clase media se debieron a cambios en el número de perceptores de ingreso por hogar entre la onda 5 y la 6 . El número de quienes reciben 
ingresos en la familia debería cambiar con las condiciones macroeconómicas, ya que más adultos encuentran empleo y los jóvenes pueden encontrar trabajos de tiempo parcial; ambos cambios aumentarán el ingreso familiar. Este análisis encontró un muy pequeño incremento en el tamaño de la clase media en Luxemburgo y una pequeña declinación en la clase media de Taiwan, debido a incrementos en el número de quienes reciben ingresos, pero en general, hubo pocos cambios atribuibles al número de perceptores por familia en cada país.

Como las nuevas series de datos del eli para Latinoamérica proporcionan sólo datos esporádicos (puntuales), carecemos de datos de series de tiempo necesarios para repetir tal análisis. No obstante, podemos examinar los cambios en el tamaño de la clase media, de acuerdo con cambios en el número de perceptores en la familia, en un país y en un determinado momento. De nuevo, las condiciones macroeconómicas deberían ser una razón de que el número de perceptores variara de país a país, y más perceptores deberían incrementar el tamaño de la clase media, especialmente porque (como vimos anteriormente) la política de impuesto y gasto gubernamental ejerce poca influencia sobre el tamaño de la clase media.

El Cuadro 6 calcula el tamaño de la clase media en las naciones latinoamericanas, desglosadas por el número de quienes reciben ingresos en la familia. En las naciones desarrolladas, tal variación es pequeña. Algunos de nuestros seis países latinoamericanos siguen este patrón. Por ejemplo, hay poca tendencia en Uruguay o Guatemala (hasta que llegamos al punto en el cual los hogares tienen más de tres perceptores, lo que posiblemente se deba al pequeño tamaño de la muestra). Por otra parte, en Brasil y México es muy claro que un incremento en el número de perceptores en el hogar trae consigo una mayor probabilidad de ingresos de clase media, y así parece que la creciente participación de los integrantes

Cuadro 6. Familias de clase media por número de miembros de la familia que reciben ingresos

\begin{tabular}{lllllc} 
& \multicolumn{5}{c}{ Número de miembros de la familia que reciben ingresos } \\
\cline { 2 - 6 } \multicolumn{1}{c}{ País } & Cero & Uno & Dos & Tres & Más de tres \\
\hline Brasil & $15.7 \%$ & $15.1 \%$ & $22.2 \%$ & $26.3 \%$ & $27.4 \%$ \\
Colombia & $13.4 \%$ & $22.6 \%$ & $23.4 \%$ & $31.4 \%$ & $19.1 \%$ \\
Guatemala & $20.1 \%$ & $18.1 \%$ & $18.4 \%$ & $19.4 \%$ & $25.0 \%$ \\
México & $14.4 \%$ & $17.0 \%$ & $21.6 \%$ & $24.4 \%$ & $31.8 \%$ \\
Perú & $18.5 \%$ & $17.9 \%$ & $20.6 \%$ & $22.2 \%$ & $23.3 \%$ \\
Uruguay & $30.4 \%$ & $23.6 \%$ & $26.7 \%$ & $31.3 \%$ & $28.4 \%$ \\
Promedios no & $18.8 \%$ & $19.1 \%$ & $22.2 \%$ & $25.8 \%$ & $25.8 \%$ \\
ponderados & & & & & \\
\hline
\end{tabular}

Fuente: Cómputos del autor utilizando la base de datos del ell. 
de un hogar en la fuerza de trabajo, es importante para generar estatus de clase media. Los resultados para Perú también indican que el número de perceptores es probablemente un factor que afecta allí el tamaño de la clase media.

Estos resultados son más notables debido a la relación entre tamaño de la familia y estatus de clase media. El ingreso adicional necesita de más miembros de la familia. Para considerar esto, el Cuadro 7 presenta dos tipos fijos de familias y examina cómo la probabilidad de ser clase media cambia al modificarse el número de quienes perciben ingresos. Muestra cómo la proporción de la clase media cambia con el número de perceptores para las parejas casadas, no viejas, con dos y con tres hijos. Los datos para las familias sin perceptores no se incluyen por el pequeńo número de familias en esa situación.

Cuadro 7. Familias de clase media por número de miembros que reciben ingresos y por características familiares seleccionadas

\begin{tabular}{|c|c|c|c|c|}
\hline \multicolumn{5}{|c|}{ Familias de casados con dos hijos, no encabezadas por alguien de edad avanzada } \\
\hline \multirow[t]{2}{*}{ País } & \multicolumn{4}{|c|}{ Número de miembros que reciben ingresos } \\
\hline & 1 & 2 & 3 & más de 3 \\
\hline Brasil ${ }^{\star}$ & $9.9 \%$ & $17.7 \%$ & $24.3 \%$ & $27.9 \%$ \\
\hline Colombia & $29.8 \%$ & $20.8 \%$ & $21.0 \%$ & $9.1 \%$ \\
\hline Guatemala & $20.4 \%$ & $18.2 \%$ & $16.2 \%$ & $11.9 \%$ \\
\hline México & $17.3 \%$ & $21.4 \%$ & $29.3 \%$ & $46.0 \%$ \\
\hline Perú & $20.9 \%$ & $20.0 \%$ & $21.1 \%$ & $24.4 \%$ \\
\hline Uruguay & $19.3 \%$ & $27.5 \%$ & $44.0 \%$ & $18.0 \%$ \\
\hline Promedios no ponderados & $19.6 \%$ & $20.9 \%$ & $26.0 \%$ & $22.9 \%$ \\
\hline \multicolumn{5}{|c|}{ Familias de casados con tres hijos, no encabezadas por alguien de edad avanzada } \\
\hline \multirow[t]{2}{*}{ País } & \multicolumn{4}{|c|}{ Número de miembros que reciben ingresos } \\
\hline & 1 & 2 & 3 & más de 3 \\
\hline Brasil ${ }^{\star}$ & $4.7 \%$ & $9.8 \%$ & $12.2 \%$ & $17.3 \%$ \\
\hline Colombia & $15.2 \%$ & $22.0 \%$ & $50.8 \%$ & $12.6 \%$ \\
\hline Guatemala & $20.6 \%$ & $24.0 \%$ & $24.4 \%$ & $31.5 \%$ \\
\hline México & $17.3 \%$ & $21.4 \%$ & $29.3 \%$ & $46.0 \%$ \\
\hline Perú & $19.6 \%$ & $27.3 \%$ & $23.8 \%$ & $38.2 \%$ \\
\hline Uruguay & $15.3 \%$ & $19.3 \%$ & $35.1 \%$ & N.A. \\
\hline Promedios no ponderados & $15.5 \%$ & $20.6 \%$ & $29.3 \%$ & $29.1 \%$ \\
\hline
\end{tabular}

Fuente: Cómputos del autor utilizando la base de datos del Ell.

Nota: no de edad avanzada significa familias encabezadas por alguien de menos de 60 años.

* Para Brasil, se dispone de datos sólo para parejas, que pueden o no estar casadas. 
De nueva cuenta, nuestros resultados son un tanto mixtos, pero difieren algo del resultado del Cuadro 6. En Uruguay, cuando el número de perceptores se incrementa de uno a tres miembros de la familia, la probabilidad de ser clase media crece acentuadamente. En Guatemala hay poco cambio: un pequeño decremento en la probabilidad de ser clase media, cuando el número de perceptores se incrementa. Perú también parece seguir este patrón. Finalmente, en Brasil y México, más perceptores incrementan la probabilidad de ser clase media. De este modo, en estos dos países parece que las variables del mercado laboral (como empleo y tasas de participación en la fuerza de trabajo y empleo) importan para obtener estatus de clase media. En los otros países latinoamericanos, en el mejor de los casos, la relación es ambigua.

Podemos examinar esta cuestión considerando algunas variables de la fuerza de trabajo en las series de datos del ELI; por ejemplo, el número de horas trabajadas por los jefes de los hogares y por los cónyuges, el número de semanas trabajadas de tiempo completo y de tiempo parcial por los jefes de los hogares y por los cónyuges, o el número de semanas que el jefe del hogar y el cónyuge estuvieron desempleados durante el año. En épocas de desempleo, esperaríamos ver declinar el número de horas trabajadas y el número de semanas trabajadas de tiempo completo durante el año. Como resultado, la clase media se achicaría. Desgraciadamente, los datos disponibles de la fuerza de trabajo son limitados en las naciones desarrolladas y lo son aún más en los países en desarrollo.

No obstante, es posible iniciar un análisis de este tipo. El Cuadro 8 examina el tamaño de la clase media por el estatus de empleo del jefe del hogar y del

Cuadro 8. Situación de clase media por estatus de empleo de parejas casadas de edad no avanzada

\begin{tabular}{lcccc} 
& \multicolumn{4}{c}{ Porcentaje de clase media cuando tienen empleo: } \\
\cline { 2 - 5 } \multicolumn{1}{c}{ País } & El jefe de familia & La esposa & Cualquiera de los dos & Los dos \\
\hline Brasil & $17.9 \%$ & $20.6 \%$ & $17.7 \%$ & $21.2 \%$ \\
Colombia & $24.7 \%$ & $20.4 \%$ & $24.3 \%$ & $20.8 \%$ \\
Guatemala & $18.7 \%$ & $18.0 \%$ & $18.7 \%$ & $17.9 \%$ \\
México & $20.7 \%$ & $23.6 \%$ & $20.6 \%$ & $24.0 \%$ \\
Perú & $19.2 \%$ & $17.0 \%$ & $19.3 \%$ & $16.8 \%$ \\
Uruguay & $25.3 \%$ & $26.9 \%$ & $25.5 \%$ & $26.7 \%$ \\
Promedios no ponderados & $21.1 \%$ & $21.1 \%$ & $21.0 \%$ & $21.2 \%$ \\
Canadá & $37.5 \%$ & $35.2 \%$ & $37.0 \%$ & $37.5 \%$ \\
EUA & $32.3 \%$ & $32.5 \%$ & $31.9 \%$ & $33.1 \%$ \\
\hline
\end{tabular}

Fuente: Cálculos del autor utlizando la base de datos del ell.

Nota: no de edad avanzada significa que el jefe de familia tiene menos de 60 años.

*Para Brasil sólo se dispone de datos por parejas, que pueden o no estar casadas. 
cónyuge. Por obvias razones consideramos sólo los hogares encabezados por alguien menor de 60 años y restringimos el análisis a parejas casadas. Calculamos y reportamos la probabilidad de que un hogar sea de clase media cuando quien lo encabeza está empleado, cuando el cónyuge está empleado, cuando alguno está empleado y cuando ambos están empleados.

El Cuadro 8 indica que el empleo parece influir poco en las naciones latinoamericanas. La probabilidad de ser de clase media no parece depender de cuál cónyuge esté empleado; es muy semejante cualquiera que sea el que trabaja. Además, la probabilidad de ser de clase media no cambia mucho cuando además del jefe de familia, trabaja el cónyuge. Yendo de la columna 1 (donde sólo el jefe de familia está empleado) a la columna 4 (donde ambos cónyuges están empleados), vemos que el tamaño de la clase media cae en Colombia, Guatemala y Perú, y se incrementa un poco en Uruguay. Sólo en Brasil y México el hecho de que el cónyuge trabaje incrementa el tamaño de la clase media nacional. Esto apoya nuestro anterior resultado acerca de la importancia del empleo en el estatus de clase media en Brasil y México.

Finalmente, en la parte inferior del Cuadro 8 añadimos cifras comparables para las dos naciones desarrolladas de América. Al igual que en las naciones latinoamericanas (con excepción de Brasil y México), en Canadá y euA no importa mucho que trabaje la esposa del jefe de familia, en cuanto a proporcionar ingresos de clase media.

\section{RESUMEN Y CONCLUSIONES}

Este estudio ha utilizado el eli para examinar el tamaño de la clase media en varios países latinoamericanos. Uno de los principales hallazgos es que a mediados de la primera década del siglo Xxi el tamaño de la clase media en nuestra muestra de países latinoamericanos no parecía depender de factores demográficos. Un segundo hallazgo es que, en contraste con la mayoría de las naciones desarrolladas, las políticas gubernamentales de impuestos y gasto influyen poco en el incremento del tamaño de la clase media en los países latinoamericanos. Finalmente, al igual que en el mundo desarrollado, los factores del mercado laboral no afectan mayor cosa el tamaño de la clase media. Las principales excepciones parecen ser Brasil y México, donde el empleo parece incrementar el tamaño de la clase media.

Estos hallazgos apoyan mi anterior trabajo (Pressman, 2007, 2010) sobre los determinantes del tamaño de la clase media, principalmente en las naciones desarrolladas. Allí se encontró que ni las variables socio-demográficas, ni las variables del mercado de trabajo fueron importantes al determinar el tamaño relativo 
de la clase media en los diferentes países. Lo que es realmente importante para que exista una gran clase media, es la política gubernamental: un sistema fiscal progresivo y una serie de generosos programas de gasto gubernamental que beneficien a las familias de ingreso bajo y medio. El carecer de esas políticas explica en buena medida el hecho de que las naciones latinoamericanas no tengan una gran clase media comparada con la de las naciones desarrolladas. Para que aumente la clase media latinoamericana, será necesario que el Estado desarrolle y expanda políticas que apoyen la existencia de una gran clase media: asignaciones infantiles y permisos familiares, seguros de desempleo y de incapacidad y un sistema de jubilación más inclusivo y generoso.

La conclusión de este documento también apoya los resultados de numerosos estudios sobre la inequitativa distribución del ingreso en el mundo. Uno de los principales hallazgos de esta vasta literatura es que generosas redes de seguridad pública y de servicios sociales se traducen en mayor equidad en el ingreso (Bradly et al, 2003; Moller et al, 2003; Western \& Healy, 1993). Aunque este trabajo se ha enfocado principalmente en naciones desarrolladas, al parecer, las mismas conclusiones son válidas en los países en desarrollo.

\section{REFERENCIAS}

Adelman, Irma \& Cynthia Morris, Society, Politics, and Economic Development: A Quantitative Approach, Baltimore, Johns Hopkins University Press, 1967. Aristotle, Politics, trans. H. Rackham, Cambridge, MA, Harvard University Press, 1932.

Barro, Robert, "Determinants of Democracy," Journal of Political Economy, vol. 107, December 1999, pp. S158-S183.

Birdsall, Nancy, Carol Graham \& Stefano Pettinato, "Stuck in the Tunnel: is Globalization Muddling the Middle Class?, Brookings Institution, Center on Social and Economic Dynamics, Working Paper N. 14, 2000.

Bradley, David, Evelyne Huber, Stephanie Moller, François Nielsen, \& John Stephens, "Distribution and Redistribution in Postindustrial Democracies," World Politics, Vol. 55, January 2003, pp. 193-228.

Burtless, Gary, "Effect of Growing Wage Disparities and Family Composition Shifts on the Distribution of U.S. Income," European Economic Review, vol. 43, April 1999, pp. 853-865.

Cancian, Maria \& Deborah Reed, "Assessing the Effect of Wives' Earnings on Family Income Inequality", Review of Economics and Statistics, vol. 80, February 1998, pp. 73-79. 
Cancian, Maria \& Deborah Reed, "Changes in Family Structure: Implications for Poverty and Related Policy," in Danziger, Sheldon \& Robert Haveman, (Eds) Understanding Poverty, New York, Russell Sage, 2001, pp. 69-96.

Cancian, Maria \& Robert Schoeni, "Wives' Earnings and the Level and Distribution of Married Couples' Earnings in Developed Countries," Journal of Income Distribution, vol. 8, Summer 1998, pp. 45-61.

Cashell, Brian, "Who are the 'Middle Class'?," Congressional Research Service Report N. RS22627, October 22, 2008.

Easterlin, Richard, "Income and Happiness: Towards a Unified Theory, Economic Journal, vol. 111, July 2001, pp. 465-484.

Easterly, William, "The Middle Class Consensus and Economic Development", Journal of Economic Growth, vol. 6, December 2001, pp. 317-335.

eClac (Economic Commission for Latin America and the Caribbean), Social Panorama of Latin America, 2005, Santiago, United Nations, 2005.

Ermisch, John, "Econometric Analysis of Birth Rate Dynamics in Britain," Journal of Human Resources, vol. 23, Fall 1988a, pp. 53-76.

Ermisch, John, "Economic Influences on Birth Rate," National Institute Economic Review, November 1988b, pp. 71-81.

Estache, Antonio \& Danny Leipziger, (Eds.) Stuck in the Middle, Washington, DC, Brookings Institution, 2009.

Esping-Andersen, Gøsta, "Sociological Explanations of Changing Income Distributions," American Behavioral Scientist, vol. 50, January 2007, pp. 639-658.

Frank, Robert, Falling Behind: How Rising Inequality Harms the Middle Class, Berkeley, CA, University of California Press, 2007.

Fuchs, Victor, "Redefining Poverty and Redistributing Income," The Public Interest, N. 8, Summer 1967, pp. 88-94.

Galbraith, James, "A Perfect Crime: Global Inequality," Deadalus, vol. 131, Winter 2002, pp. 11-25.

Gauthier, Anne-Helene \& Jan Hatzius, "Family Benefits and Fertility: An Econometric Analysis," Population Studies, vol. 51, November 1997, pp. 295-306.

Goldstein, Markus \& Estache, Antonio, "The Scope and Limits of Subsidies," in Estache, Antonio \& Leipziger, Danny (Eds), Stuck in the Middle, Washington, DC, Brookings Institution, 2009, pp. 75-96.

Gottschalk, Peter \& Sheldon Danziger, "Inequality of Wage Rates, Earnings and Family Income in the United States, 1975-2002," Review of Income and Wealth, vol. 51, June 2005, pp. 231-254. 
Horrigan, Michael \& Steven Haugen, "The Declining Middle Class Thesis: A Sensitivity Analysis, Monthly Labor Review, vol. 111, N. 5, May 1988, pp. 3-13. Karoly, Lynn \& Gary Burtless, "The Effects of Rising Earning Inequality on the Distribution of us Income," Demography, vol. 32, August 1995, pp. 379-405.

Korzeniewicz, Roberto \& William Smith, "Poverty, Inequality and Growth in Latin America: Searching for the High Road to Globalization," Latin America Research Review, vol. 35, October 2000, pp. 7-54.

Landes, David, The Wealth and Poverty of Nations, New York, W.W. Norton, 1998.

Layard, Richard, Happiness: Lessons from a New Science, New York, Penguin Press, 2005.

Lerman, Robert \& Shlomo Yitzhaki, "Income Inequality Effects by Income Sources: A New Approach and Applications to the United States", Review of Economics and Statistics, vol. 67, February 1985, pp. 151-156.

Lindert, Kathy, Emmanuel Skoufias, \& Joseph Shapiro, Redistributing Income to the Poor and the Rich: Public Transfers in Latin America and the Caribbean, Washington, DC, World Bank, 2006.

Luttmer, Erzo, "Neighbors as Negatives: Relative Earnings and Well-being," Quarterly Journal of Economics, vol. 120, August 2005, pp. 963-1002.

Luxembourg Income Study (LIS) Database, http://www.lisproject.org/techdoc.htm (multiple countries; accessed 28 August 2009 through 28 August 2010).

Macinol, John, The Movement for Family Allowances, 1918-45: A Study in Social Policy Development, London, Heinemann, 1980.

Malthus, Thomas Robert, An Essay on the Principles of Population, $2^{\text {nd }}$ ed., London, 1803.

Moller, Stephanie, Evelyne Huber, John Stephens, David Bradley, \& François Nielsen, "Determinants of Relative Poverty in Advanced Capitalist Democracies", American Sociological Review, vol. 68, February 2003, pp. 22-51. OECD, The OECD List of Social Indicators, Paris, OECD, 1982.

Orshansky, Mollie, "Consumption, Work and Poverty," in Seligman, B.B. (Ed.) Poverty as a Public Issue, New York, Free Press, 1965, pp. 52-84.

Orshansky, Mollie, "How Poverty is Measured," Monthly Labor Review, vol. 92, N. 2, February 1969, pp. 26-41.

Pew Research Center, Inside the Middle Class: Bad Times Hit the Good Life, Washington, DC, Pew Research Center, 2008.

Pressman, Steven, "The Decline of the Middle Class: An International Perspective," Journal of Economic Issues, vol. 41, March 2007, pp. 181-200. 
Pressman, Steven, "Public Policy and the Middle Class in the Mid 2000s", Journal of Economic Issues, vol. 44, March 2010, pp. 243-262.

Sen, Amartya, Development as Freedom, New York, Random House, 1999.

Shorrocks, Anthony, "The Impact of Income Components on the Distribution of Family Income," Quarterly Journal of Economics, vol. 98, May 1983, pp. 311-326.

Smeeding, Timothy, Brigitte Buhmann \& Lee Rainwater, "Equivalence Scales, Well-Being, Inequality and Poverty: International Comparisons Across Ten Countries Using the Luxembourg Income Study (LIS) Database," LIS-CEPS Working Paper N. 17, 1988.

Smeeding, Timothy, Lee Rainwater, B. Buhmann, \& Gunther Schmaus, "Luxembourg Income Study (LIS) Information Guide," LIS-CEPS Working Paper N. 8, 1988.

Smeeding, Timothy, Gunther Schmaus \& Serge Allegra, "An Introduction to LIS," LIS-CEPS Working Paper N. 1, 1985.

Thurow, Lester, "The Disappearance of the Middle Class," New York Times, Feb. 5, 1985, p. F3.

Vadakin, James, Family Allowances, Miami, University of Miami Press, 1958.

Vadakin, James, Children, Poverty, and Family Allowances, New York, Basic Books, 1968.

Western, Bruce \& Kieran Healy, "Explaining the oECD Wage Slowdown: Recession or Labor Decline?," European Sociological Review, vol. 15, N. 3, September 1999, pp. 233-249.

Apéndice

\begin{tabular}{ll}
\hline \multicolumn{1}{c}{ País } & \multicolumn{1}{c}{ Fuente } \\
\hline Brasil & National Household Sample Survey / Pesquisa Nacional por Amostra de Domicilios (PNAD) \\
Colombia & Continuous Household Survey / Encuesta Continua de Hogares (ECH) \\
Guatemala & National Survey of Living Conditions / Encuesta Nacional de Condiciones de Vida (ENCOVI) \\
México & Household Income and Expenditure Survey, 2000 and 2004 \\
Perú & National Household Survey / Encuesta Nacional de Hogares (ENAHO) \\
Uruguay & Continuous Household Survey / Encuesta Continua de Hogares (ECH) \\
\hline
\end{tabular}

